\title{
Charged-particle heating in imbalanced MHD turbulence
}

\author{
Martin S. WeidI* \\ Max-Planck/Princeton Research Center for Plasma Physics and Max-Planck-Institut für \\ Plasmaphysik, Boltzmannstr. 2, 85748 Garching, Germany \\ E-mail: martin.weidleipp.mpg.de
}

\section{Bogdan Teaca}

Applied Mathematics Research Centre, Coventry University, Coventry CVI 5FB, UK

\section{Frank Jenko}

Max-Planck/Princeton Research Center for Plasma Physics and Max-Planck-Institut für Plasmaphysik, Boltzmannstr. 2, 85748 Garching, Germany

\section{Reinhard Schlickeiser}

Institut für Theoretische Physik, Lehrstuhl IV: Weltraum- und Astrophysik, Ruhr-Universität Bochum, 44780 Bochum, Germany

\begin{abstract}
Astrophysical plasmas such as the solar wind often exhibit characteristics of imbalanced magnetohydrodynamic (MHD) turbulence, in which the fluctuations of the bulk velocity and the magnetic field are strongly correlated. As a result, turbulent heating is less efficient than in the more commonly studied case of balanced turbulence.

We study the transport and acceleration properties of charged particles in imbalanced MHD turbulence by performing test-particle simulations. The cross-helicity level, measuring the degree of imbalance of the MHD steady-state, is controlled by using a correlated forcing scheme for velocity and magnetic fields. We discuss the decrease of the turbulent heating rate in systems with non-zero cross-helicity and compare its scaling with theoretical predictions, and show the pitchangle asymmetry of the scattering coefficient in cross-helical turbulence. Our results are relevant for any plasma in which turbulent heating is important, for example the heating of dust particles in the interstellar medium or the injection of thermal protons into the Fermi-II acceleration process in supernova remnant shocks.
\end{abstract}

Cosmic Rays and the InterStellar Medium - CRISM 2014,

24-27 June 2014

Montpellier, France

${ }^{*}$ Speaker. 


\section{Introduction}

Magnetohydrodynamic turbulence in astrophysical systems is often cross-helical, i.e. the fluctuations of velocity and magnetic fields are strongly correlated. This property, also known as imbalanced turbulence, is well-known from observations of the solar wind [1] and has been predicted to be important in the upstream medium of supernova remnant shocks [2] as well as a driving force of the dynamo effect in accretion disks and young galaxies [3, 4]. Although the influence of cross helicity on the rate at which turbulent energy is dissipated and transported has been the subject of many discussions in the astrophysical community [5,6], the effect which imbalanced turbulence has on the transport and heating of charged particles in the interstellar medium has been only seldomly investigated in simulations. A notable exception is [7], who performed test-particle simulations in static snapshots of MHD turbulence and found that spatial diffusion of ultra-relativistic cosmic-ray particles was not affected by varying the cross helicity. More generally it was conjectured in [8] that the degree of imbalance would barely change the heating rate of charged particles, in apparent contradiction to earlier results by $[9,10]$, who had used quasilinear diffusion theory to calculate the dependence of pitch-angle scattering and the heating rate on cross helicity. In the following we present simulations of cross-helical MHD turbulence and show how test-particle transport and heating are affected by the level of imbalance.

\section{Theory}

\subsection{MHD theory}

We use the pseudospectral code TURBO [11] to solve the equations of incompressible resistive forced magnetohydrodynamics (MHD):

$$
\begin{aligned}
& \frac{\partial \mathbf{u}}{\partial t}=-(\mathbf{u} \circ \nabla) \mathbf{u}+(\mathbf{b} \circ \nabla) \mathbf{u}+v \nabla^{2} \mathbf{u}+\mathbf{f}_{u}-\nabla \tilde{p}, \\
& \frac{\partial \mathbf{b}}{\partial t}=-(\mathbf{u} \circ \nabla) \mathbf{b}+(\mathbf{b} \circ \nabla) \mathbf{b}+\eta \nabla^{2} \mathbf{b}+\mathbf{f}_{b},
\end{aligned}
$$

where $\mathbf{u}$ and $\mathbf{b}$ are the velocity and the magnetic field (in Alfvén units), respectively, $v$ and $\eta$ denote the kinematic viscosity and the resistivity, and $\mathbf{f}_{u}$ and $\mathbf{f}_{b}$ are external forces acting on the velocity and magnetic fields. The pressure term $\nabla \tilde{p}$ is accounted for by imposing $\nabla \circ \mathbf{u}=0$. All simulations are performed in the plasma rest frame $(\langle\mathbf{u}\rangle=0)$ on cubic grids with a resolution of $512^{3}$ and a physical size of $2 \pi$ in all three dimensions, and we compare runs without a magnetic mean-field to others with a constant magnetic mean-field in $z$-direction. The dissipation coefficients $v$ and $\eta$ are chosen to be equal and sufficiently small that Kolmogorov's dissipation length $\ell_{K}=\left(v^{3} / \varepsilon_{\text {inj }}\right)^{1 / 4}$ is well resolved, where $\varepsilon_{\text {inj }}$ is the energy injection rate (cf. [12]).

In ideal MHD without external forcing, such that $v=\eta=0$ and $\mathbf{f}_{u}=\mathbf{f}_{b}=0$, three invariant quantities are conserved in a closed system: the energy $\mathscr{E}=\left\langle\mathbf{u}^{2}+\mathbf{b}^{2}\right\rangle / 2$, the cross helicity $\mathscr{K}=$ $\langle\mathbf{u} \circ \mathbf{b}\rangle$, and the magnetic helicity $\mathscr{H}_{\text {mag }}=\langle\mathbf{A} \circ \mathbf{b}\rangle$, where $\mathbf{A}$ is the magnetic vector potential and $\langle\cdot\rangle$ denotes averaging over a closed volume. The close relation between cross helicity and Alfvén waves becomes obvious when the former is expressed as $\mathscr{K}=\mathscr{E}^{+}-\mathscr{E}^{-}$, with the Elsasser energies $\mathscr{E}^{ \pm}=\langle\mathbf{u} \pm \mathbf{b}\rangle^{2} / 4$ measuring the alignment or anti-alignment of velocity and magnetic field in the 
system. In the presence of a magnetic mean field $\langle\mathbf{b}\rangle=\mathbf{B}_{0}$ along the $z$ axis, shear Alfvén waves will propagate at the Alfvén velocity $v_{A}=\left\langle\mathbf{b}^{2}\right\rangle^{1 / 2}$ along and opposite $\mathbf{B}_{0}$, and the Elsasser energies indicate how the turbulent energy is distributed among co- and counter-propagating waves: A cross helicity of $\mathscr{K}=0$ implies energy equipartition of waves in both direction, whereas the absence of Alfvén waves in either direction leads to a normalized cross helicity $\sigma^{c}=\left(\mathscr{E}^{+}-\mathscr{E}^{-}\right) /\left\langle\left(\mathbf{u}^{2}+\right.\right.$ $\left.\left.\delta \mathbf{b}^{2}\right) / 4\right\rangle$ of $\sigma^{c}= \pm 1$.

In order to control the level of cross helicity in our simulations, we start with random initial conditions (such that $\mathscr{K}(0)=\mathscr{H}_{\text {mag }}(0)=0$ ) and drive the system by injecting energy into all modes with wave vectors in the interval $2.5<k<3.5$. The forcing algorithm determines the amplitudes of $\mathbf{u}$ and $\mathbf{b}$ modes of left- and right-handed circular polarization separately and then adjusts the forcing amplitudes of each mode [13] so that the total energy injection rate $\varepsilon_{\text {inj }}$ and the cross-helicity injection rate $\sigma_{\text {inj }}$ are constant over time, while the magnetic helicity $\mathscr{H}_{\text {mag }}$ is kept vanishingly small.

\subsection{Quasilinear theory}

The stochastic evolution of a distribution of charged particles in MHD turbulence with a magnetic mean-field is generally described by the Fokker-Planck equation [14]

$$
\frac{\partial f}{\partial t}=\frac{\partial}{\partial \mu} D_{\mu \mu} \frac{\partial}{\partial \mu} f+\frac{\partial}{\partial \mu} D_{\mu p} \frac{\partial}{\partial p} f+p^{-2} \frac{\partial}{\partial p} p^{2}\left(D_{\mu p} \frac{\partial}{\partial \mu} f+D_{p p} \frac{\partial}{\partial p} f\right),
$$

where $f(\mu, p ; t)$ is the distribution of particles in the phase-space spanned by the pitch-angle cosine $\mu=v_{z} / v$ and the momentum $p$. The diffusion coefficients $D_{\mu \mu}, D_{\mu p}$, and $D_{p p}$ depend on the properties of the specific turbulence realization and in particular on the normalized cross helicity $\sigma^{c}$. In the simple case of isospectral slab turbulence with a spectral density $P(k) \propto k_{z}^{-s}$, the momentum diffusion coefficient for an isotropic charged-particle distribution is [10]

$$
\bar{D}_{p p}=\pi \omega_{g} p^{2} \frac{v_{A}^{2}}{v^{2}} \frac{s-1}{s(s+2)} \frac{\left\langle\delta \mathbf{b}^{2}\right\rangle}{\mathbf{B}_{0}^{2}}\left(r_{g} k_{\min }\right)^{s-1}\left(1-\sigma^{c 2}\right)
$$

where $\omega_{g}=q\left|\mathbf{B}_{0}\right|$ is the gyrofrequency of the particles with charge-to-mass ratio $q$ and $k_{\min }=$ $2 \pi / L_{\mathrm{inj}}$ is the wave number corresponding to the correlation length of the turbulence.

\section{Results}

\subsection{MHD results}

Before we inject test-particles into the turbulence runs, we allow each simulation first to settle into a steady-state as characterized by an approximately constant energy $\mathscr{E}=\left\langle\mathbf{u}^{2}+\mathbf{b}^{2}\right\rangle / 2$ and normalized cross helicity $\sigma^{c}$ (fig. 1). Depending on the rate of cross-helicity injection chosen for the forcing of each run, the steady-states exhibit different levels of normalized cross helicity $\sigma^{c}$, from balanced turbulence with $\sigma^{c}=0.0$ with zero cross-helicity injection, to strongly imbalanced MHD turbulence with $\sigma^{c} \sim 0.9$ for a cross-helicity injection of $\sigma_{\text {inj }}=0.8$. As our forcing scheme injects equal amounts of energy into modes with left- and right-handed polarization, the normalized magnetic helicity remains close to its initial value of zero in each case. 

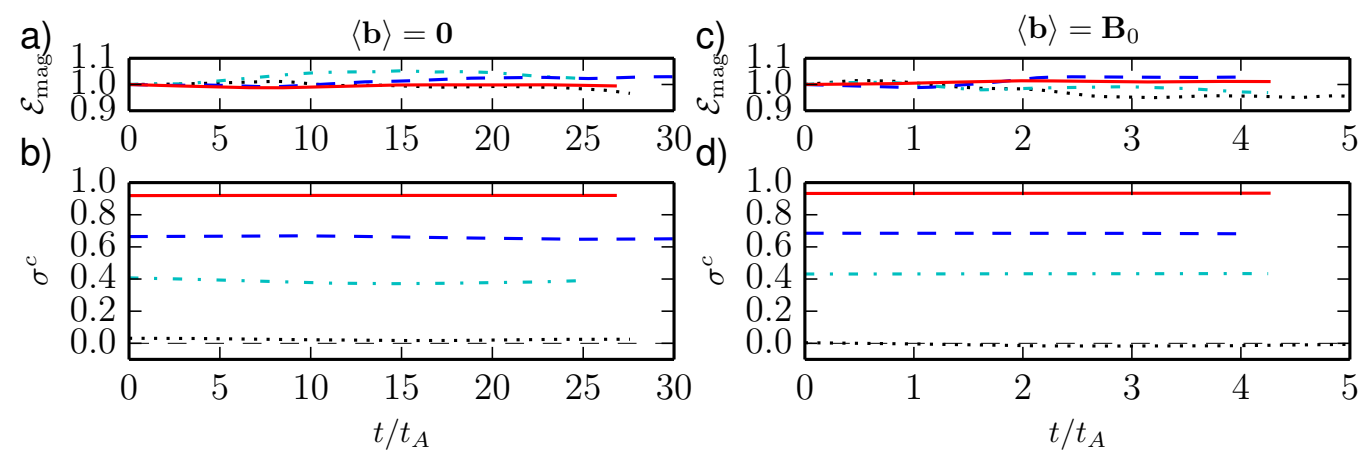

Figure 1: Evolution of magnetic energy $\mathscr{E}_{\text {mag }}=\mathbf{b}^{2} / 2$, normalized to its value at $t=0$, and normalized cross helicity $\sigma^{c}=\mathscr{K} / \mathscr{E}$ for runs a),b) without and c),d) with a order-unity magnetic mean-field, for different cross-helicity injection rates: $\sigma_{\text {inj }}=0.0$ (black, dotted), $\sigma_{\text {inj }}=0.3$ (cyan, dash-dotted), $\sigma_{\text {inj }}=0.5$ (blue, dashed), and $\sigma_{\text {inj }}=0.8$ (red, solid)

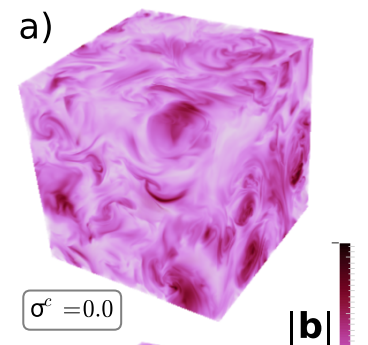

b)

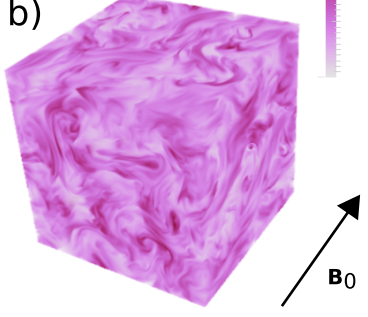

c)

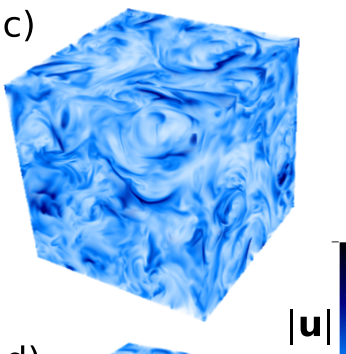

d)

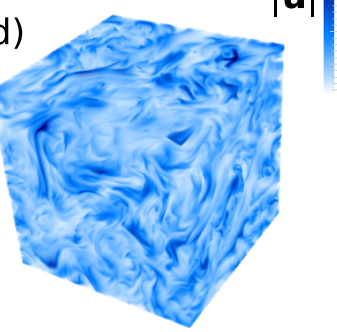

e)

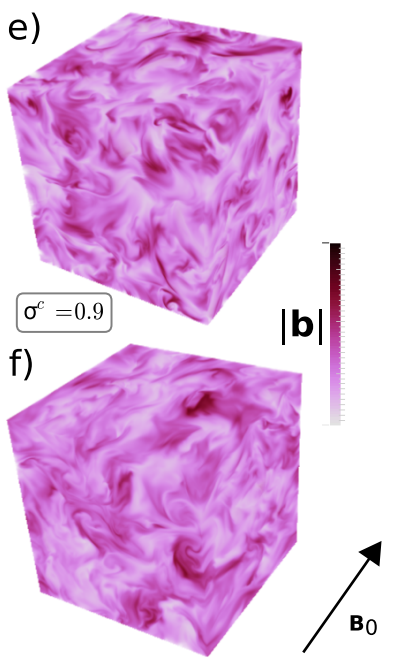

g)

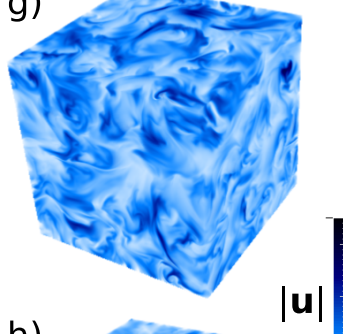

h)

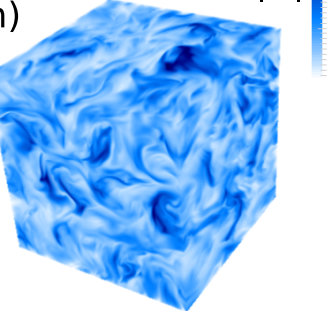

Figure 2: Spatial distribution of magnetic and velocity field intensity with zero magnetic mean-field (top row) and order-unity magnetic mean-field (bottom row). Shown are the norms of velocity $\mathbf{u}$ and magnetic field $\mathbf{b}$ in balanced turbulence (a-d) and in strongly cross-helical turbulence (e-h)

Snapshots of the norm of the velocity and the magnetic fields in the steady-states are shown in fig. 2. The presence of a magnetic mean-field $\mathbf{B}_{0}^{2} \sim \delta \mathbf{b}^{2}$ along the $z$ axis introduces a small level of anisotropy in its direction, while the turbulent fields remain isotropic in the $x-y$ plane. Even in the balanced cases $\left(\sigma^{c} \sim 0.0\right)$ regions of peak values of the magnetic field often exhibit high values of the velocity field intensity as well, so that both fields appear correlated in each pair of field plots, although the correlation is visibly more pronounced in the strongly cross-helical runs.

The role of cross helicity becomes more obvious in an analysis of the alignment of velocity and magnetic field, $\cos [\angle(\mathbf{u}, \mathbf{b})]$ (fig. 3). The cross-helical cases exhibit an increasingly positive level of alignment by definition, whereas the balanced cases contain regions of positive and negative alignment in equal measure. As observed in other simulations of balanced turbulence [15], the alignment histograms of both $\sigma^{c}=0.0$ runs are peaked at the extremal values $\cos [\angle(\mathbf{u}, \mathbf{b})]= \pm 1$. 

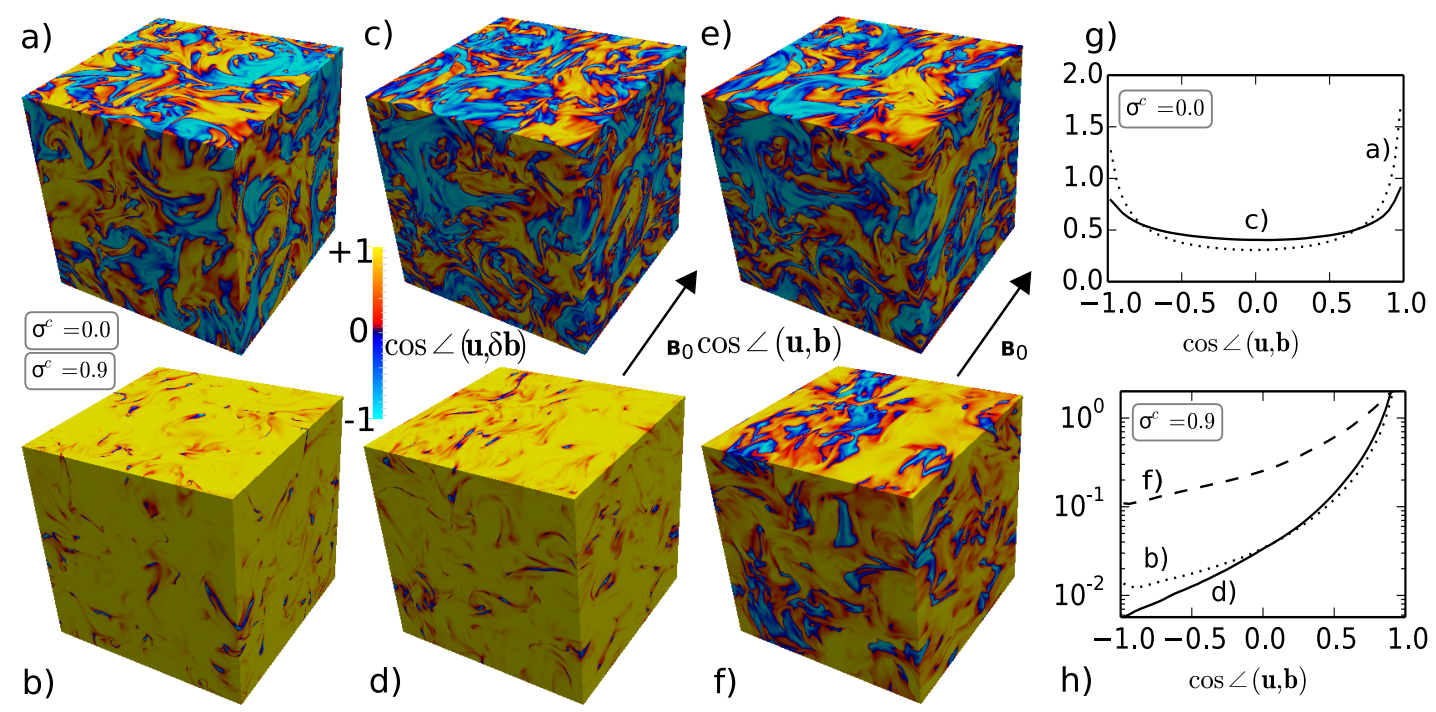

Figure 3: Alignment of velocity and magnetic fields in balanced (top row) and strongly cross-helical (bottom row) turbulence. a,b) $\cos \angle(\mathbf{u}, \mathbf{b})$ for zero-mean-field. c,d $) \cos \angle(\mathbf{u}, \delta \mathbf{b})$ for order-unity mean-field. e,f) $\cos \angle(\mathbf{u}, \mathbf{b})$ for order-unity mean-field. g,h) Histograms of distributions of $\cos \angle(\mathbf{u}, \mathbf{b})$ in zero-mean-field (dotted), and of $\cos \angle(\mathbf{u}, \mathbf{b})$ (solid) and $\cos \angle(\mathbf{u}, \delta \mathbf{b})$ (dashed) in order-unity mean-field

These peaks are less pronounced in the presence of a global magnetic mean-field, however, due to the smaller relative fluctuations of the magnetic field. In cross-helical cases, the alignment histograms of the mean-field runs considering only the fluctuations in the magnetic field, $\delta \mathbf{b}=$ $\mathbf{b}-\mathbf{B}_{0}$, are barely distinguishable from the isotropic runs without a mean-field, as one might expect from the comparable values of $\sigma^{c}$. Using an alignment definition that takes the mean-field into account yields a histogram with a less pronounced peak (dashed line in fig. $3 \mathrm{~h}$ ), since positively aligned fluctuations of $\mathbf{u}$ and $\delta \mathbf{b}$ are directed opposite to $\mathbf{B}_{0}$ in some regions.

Examining the distribution of energy in spectral space (fig. 4), we find that the balanced steady-states exhibit equipartition between positive and negative Elsasser energies $\mathscr{E}^{ \pm}$, whereas the strongly cross-helical states $\left(\sigma^{c}=0.9\right)$ show a dominance of $\mathscr{E}^{+}$over $\mathscr{E}^{-}$. The spectral slopes in the balanced cases are close to their expected values in a steady state $\left(\mathscr{E}(k) \propto k^{-3 / 2}\right.$ in the isotropic case, $\mathscr{E}\left(k_{z}\right) \propto k_{z}^{-2}$ with $\left.\mathbf{B}_{0}^{2}=\left\langle\delta \mathbf{b}^{2}\right\rangle^{1 / 2}\right)$. In the strongly imbalanced cases the slopes of positive and negative Elsasser energies differ, with the dominant positive Elsasser energy exhibiting a steeper dependence on both the perpendicular and parallel wave number with respect to the mean-field direction (cf. [16].

\subsection{Test-particle results}

We investigate the transport properties of Alfvénic $\left(v \sim v_{A}\right)$ charged particles in MHD turbulence by injecting test-particles into each simulation once energy and cross-helicity have settled in a steady-state (as shown in fig. 1). First we analyze the acceleration of charged particles by propagating 50,000 test-particles with an electric field derived from both the Ohmic electric field $\mathbf{e}_{\mathrm{ohm}}=\eta \nabla \times \mathbf{b}$ and the motional electric field $\mathbf{e}_{\mathrm{mot}}=-\mathbf{u} \times \mathbf{b}$ :

$$
\frac{\mathrm{d}}{\mathrm{d} t} \mathbf{x}=\mathbf{v}, \quad \frac{\mathrm{d}}{\mathrm{d} t} \mathbf{v}=q\left(\mathbf{e}_{\mathrm{ohm}}+\mathbf{e}_{\mathrm{mot}}+\mathbf{v} \times \mathbf{b}\right) .
$$



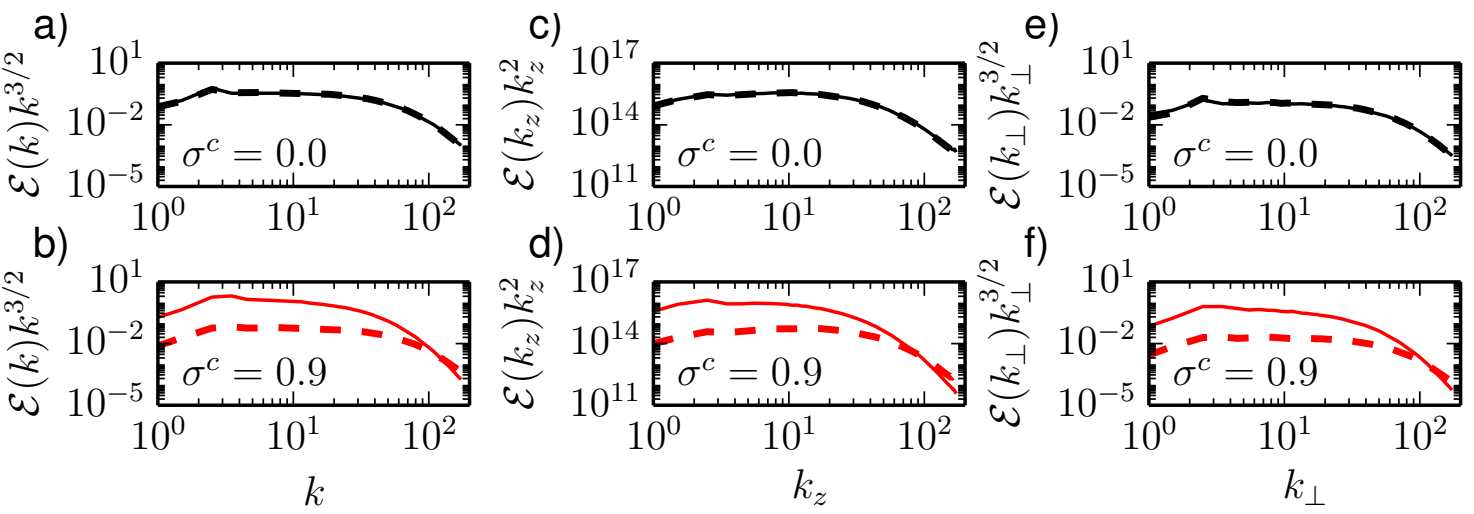

Figure 4: Spectra of positive (thin solid) and negative (thick dashed) Elsasser energies, $\mathscr{E}^{ \pm}(k)=[\mathbf{u}(k) \pm$ $\mathbf{b}(k)]^{2} / 4$, for (a) balanced turbulence and (b) strongly cross-helical turbulence with zero magnetic meanfield, and turbulence with order-unity magnetic mean-field parallel (c,d) and perpendicular (e,f) to the meanfield direction
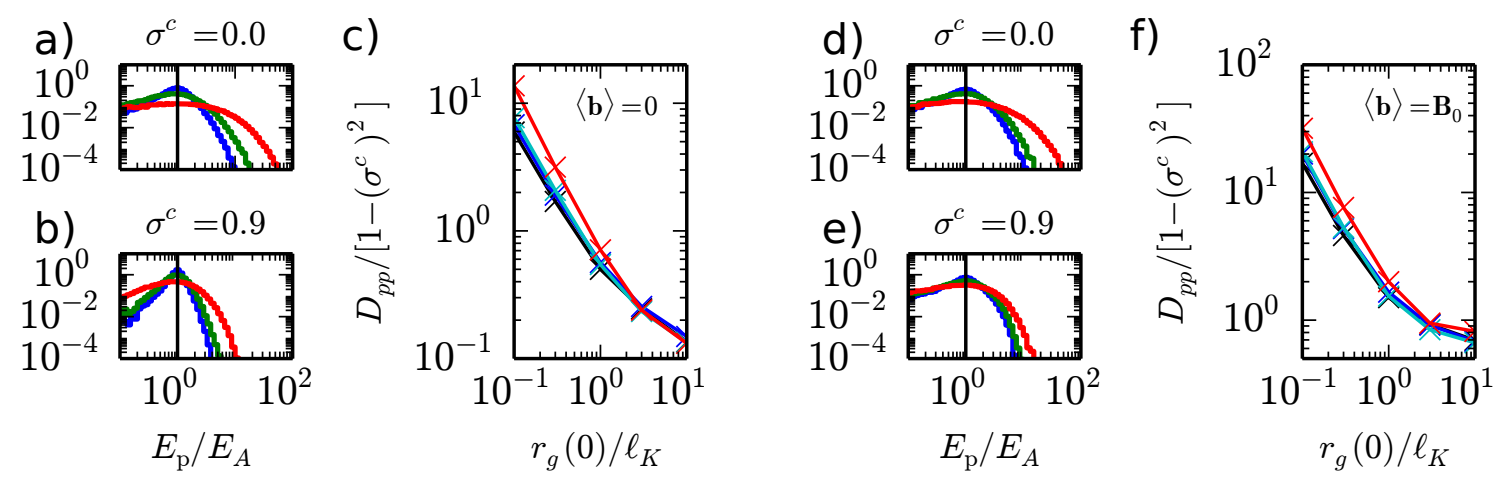

Figure 5: Energy histograms of test-particle simulations with $r_{g}(0)=3 \ell_{K}$ for zero-mean-field runs $(\mathrm{a}, \mathrm{b})$ and order-unity mean-field (d,e) in balanced and strongly cross-helical runs, at times $t / t_{A}=0$ (black), $t / t_{A}=1$ (blue), $t / t_{A}=5$ (green), and $t / t_{A}=25$ (red). Also shown are momentum pitch-angle diffusion coefficients in units of $v_{A}^{2} /\left(2 t_{A}\right)$, normalized by $\left(1-\sigma^{c 2}\right)$, for c) zero-mean-field cases and $\mathrm{f}$ ) order-unity mean-field, with cross helicities color-coded as in fig. 1

Here $q$ is the charge of the particles with unit mass, normalized such that $r_{g}(0)=v_{A} /\left(q v_{A}\right)$ corresponds to the initial gyroradius of the test-particles, which are injected with a velocity equal to the Alfvén velocity $v(0)=v_{A}$ and a randomly chosen initial position and momentum direction. Since the acceleration effect we study occurs over several Alfvén times, we evolve the test-particle trajectories in parallel with the MHD fields to simulate realistic interactions with propagating Alfvén waves.

The energy histograms (fig. 5)) of the distribution of kinetic energies $E_{p}=v^{2} / 2$ clearly show that in all cases test-particles are stochastically accelerated by the random electric fields. Comparing the effect of different turbulence configurations on ensembles with the same normalized charge, one finds the most efficient acceleration in balanced turbulence $\left(\sigma^{c}=0.0\right)$. Increasing the level of cross helicity slows down the acceleration, as we have shown in greater detail in [12].

Qualitatively speaking, of course, this diminished stochastic acceleration in cross-helical tur- 
bulence is what one expects due to the reduced norm of $\mathbf{e}_{\mathrm{mot}}=-\mathbf{u} \times \mathbf{b}$ if velocity and magnetic fields are aligned. A more quantitative analysis can be performed by computing the momentum diffusion coefficients $D_{p p}=\left\langle\left[v(\Delta t)-v_{A}\right]^{2}\right\rangle /(2 \Delta t)$ for each test-particle ensemble and rescaling them by the cross-helicity dependence as predicted by QLT (eqn. (2.4)) for a simple analytic turbulence model, $D_{p p} /\left(1-\sigma^{c 2}\right)$ (fig. 5). For gyroradii larger than the dissipation length, $r_{g}(0)>\ell_{K}$, the rescaled curves coincide, confirming that the QLT prediction is also valid in realistic MHD turbulence with three-dimensional spectra. For gyroradii comparable to or smaller than the dissipation length, however, the matching of the rescaled momentum diffusion coefficients for different cross helicities becomes increasingly worse. We attribute this effect to a dominance of Ohmic heating by $\mathbf{e}_{\mathrm{ohm}}$, the mean amplitude of which is independent of $\sigma^{c}$, for test-particles that are so strongly magnetized that their trajectories do not sample a significant amount of fluctuations of the electric field in one gyroperiod. The stochastic acceleration by $\mathbf{e}_{\text {mot }}$ is far less important for these particles, since the energy gain over one half of the gyromotion is approximately cancelled by an energy loss during the other half. Similar effects have been observed in previous simulations [17, 18].

The difference between small and large gyroradii is visible in the evolution of the squared pitch-angle cosine with respect to the mean-field, $(\cos \alpha)^{2}=v_{z}^{2} / v^{2}$, as well (fig. 6a-d). Ohmic acceleration, which mainly affects test-particles with $r_{g}(0)<\ell_{K}$, is directed parallel to the current density vector $\mathbf{j}=\nabla \times \mathbf{b}$. In the presence of a magnetic mean-field current sheets, in which the large current density accelerates particles coherently, are predominantly aligned with the meanfield. Hence Ohmic acceleration of an initally isotropic ensemble of strongly magnetized testparticles will have a net component along the $z$ direction (dashed lines in fig. 6a-d). Once particles have gained sufficient energy that their gyroradius exceeds the width of the current sheets, the net effect of Ohmic acceleration becomes less efficient and pitch-angle scattering and stochastic acceleration due to $\mathbf{e}_{\text {mot }}$ re-isotropize the momentum distribution. Since the latter effect is reduced in cross-helical turbulence, the decrease in the parallel velocity component that eventually leads to isotropization is visibly slower for $\sigma^{c} \neq 0$ than in balanced turbulence runs.

Test-particles whose initial gyroradius is larger than the dissipation length are less susceptible to Ohmic acceleration along the mean-field. On the contrary, stochastic acceleration, always perpendicular to the mean-field, is so much stronger that the kinetic energy in the perpendicular directions grows initially faster than the parallel energy and $v_{z}^{2}<v^{2} / 3$ for $r_{g}(0) \gg \ell_{K}$ (solid lines in fig. 6a-d).

Although the qualitative effect which cross helicity has on an isotropic particle distribution is minor, the picture changes for anisotropic distributions. To confirm this, we have injected ensembles of 1,000 test-particles into each of the cases with a magnetic mean-field, at random positions but with a clearly defined initial pitch-angle with respect to the $z$ direction, while keeping the initial velocity equal to the Alfvén velocity. The normalized charge is chosen such that $r_{g}(0)=2 \ell_{K}$. As we have found Ohmic acceleration to be subdominant in this regime, we neglect $\mathbf{e}_{\mathrm{ohm}}$ in the Lorentz equation for these simulations. Fig. 6e-h shows the pitch-angle diffusion coefficient $D_{\mu \mu}=\left\langle[\mu(\Delta t)-\mu(0)]^{2} /(2 \Delta t)\right.$ calculated over $\Delta t=0.01 v_{A} / r_{g}(0)$ as a function of the initial pitch-angle cosine $\mu_{0}=v_{z}(0) / v_{A}$, both for static snapshots (solid circles) and evolving MHD turbulence (crosses). In balanced-turbulence runs the graphs are symmetric, $D_{\mu \mu}(\mu) \sim D_{\mu \mu}(-\mu)$. Since scattering is enhanced by particles resonating with Alfvén waves, cross-helical turbulence, with more energy in Alfvén waves traveling in one direction with respect to the mean field than 

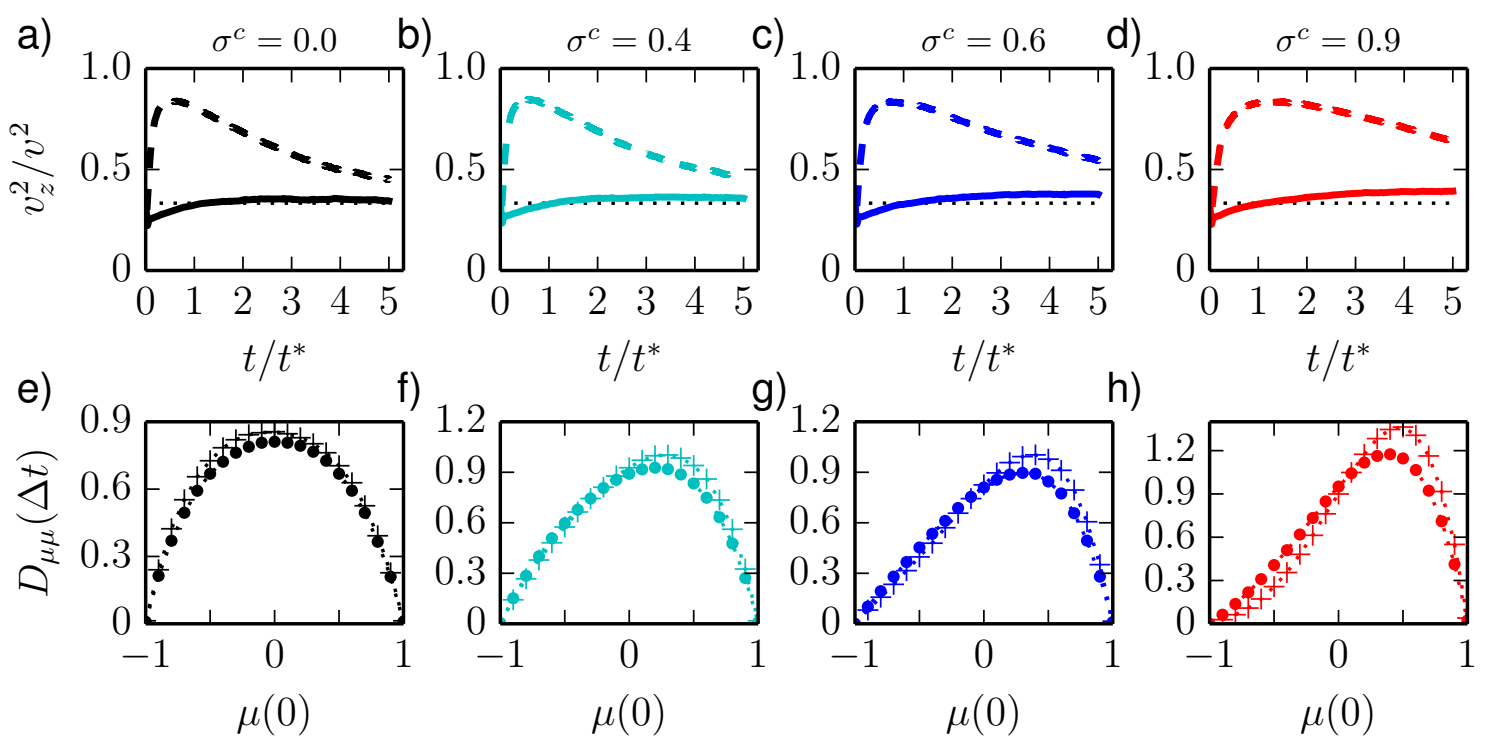

h)

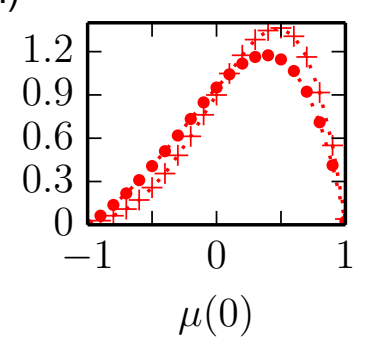

Figure 6: a)-d) Evolution of the parallel energy in units of the total kinetic energy, $v_{z}^{2} / v^{2}$, for an initially isotropic test-particle distribution with $v(0)=v_{A}$ and $r_{g}(0)=0.1 \ell_{K}$ (dashed) and $r_{g}(0)=3 \ell_{K}$ (solid), for increasing levels of cross helicity. e)-h) Pitch-angle diffusion coefficient after $\Delta t=0.01 / \omega_{g} \approx 0.01 t_{A}$, for static-MHD (dots) and evolving-MHD (crosses) runs

in the opposite direction, scatters particles with a velocity component in the mean-field direction more efficiently. Although the structure of the electric fields leads to scattering that is asymmetric with respect to the pitch-angle cosine $\mu$ even in static simulations, in which no traveling waves are present which particles could resonate with, the asymmetry is greatly enhanced if the turbulence is evolved in parallel with the particle trajectories. This is a clear indication that the asymmetric scattering we observe is indeed due to resonance with the dominating wave population, as we will further demonstrate in a forthcoming publication.

\section{Conclusions}

We have presented numerical simulations showing how the stochastic heating of charged particles is inhibited in cross-helical MHD turbulence. The results of (Dung 90) for a simple analytic turbulence model are clearly also valid for realistic three-dimensional turbulence. However, as the stochastic heating rate is a decreasing function of the particle gyroradius, we also agree with the conclusion of [7] that the degree of imbalance is not important for the turbulent transport of high-energy cosmic-ray particles.

In addition, we have presented the first numerical observations of resonant pitch-angle scattering in imbalanced turbulence. A more detailed analysis of how the pitch-angle asymmetry of scattering depends on acceleration by turbulent electric fields and the actual propagation of waves in time-dependent turbulence is currently in preparation.

\section{References}

[1] E. Marsch \& C.-Y. Tu. Journal of Geophys. Res. 95 (1990) 8211. 
[2] R. Schlickeiser \& A. Shalchi. ApJ 668 (2008) 292.

[3] A. Yoshizawa \& N. Yokoi. ApJ 407 (1993) 540.

[4] A. Brandenburg \& V. Urpin. A\&A 332 (1998) L41.

[5] Y. Zhou \& W. H. Matthaeus. Journal of Geophys. Res. 95 (1990) 10291.

[6] J. J. Podesta. Phys. of Plasmas 18 (2011) 012907.

[7] A. Beresnyak, H. Yan, A. Lazarian. ApJ 728 (2011) 60.

[8] B. D. G. Chandran, E. Quataert, G. G. Howes, J. V. Hollweg \& W. Dorland. ApJ 701 (2009) 652.

[9] R. Schlickeiser. ApJ 336 (1989) 243.

[10] R. Dung \& R. Schlickeiser. A\&A 240537.

[11] B. Teaca, M. K. Verma, B. Knaepen \& D. Carati. Phys. Rev. E 79 (2009) 46312.

[12] B. Teaca, M. S. Weidl, F. Jenko \& R. Schlickeiser. Phys. Rev. E 90 (2014) 021101(R).

[13] B. Teaca, C. C. Lalescu, B. Knaepen \& D. Carati. arXiv:1108. 2650.

[14] R. Schlickeiser. Cosmic-Ray Astrophysics (Springer, 2002).

[15] W. H. Matthaeus, A. Pouquet, P. D. Mininni, P. Dmitruk \& B. Breech. Phys. Rev. Lett. 100 (2008) 085003.

[16] R. Grappin, J. Leorat \& A. Pouqet. A\&A 126 (1983) 51.

[17] J. Ambrosiano, W. H. Matthaeus, M. L. Goldstein \& D. Plante. Journal of Geophys. Res. 93 (1988) 14383.

[18] S. Dalena, A. F. Rappazzo, P. Dmitruk, A. Greco \& W. H. Matthaeus. ApJ 783 (2014) 143. 